\title{
The Effects of Alcohol Consumption in Type 2 Diabetic Mellitus Individuals
}

\section{Addisu Dabi Wake*}

Nursing Department, College of Health Sciences, Arsi University, Asella, Ethiopia

*Corresponding author: Addisu Dabi Wake, Nursing Department, College of Health Sciences, Arsi University, P.O. Box: 393, 04 Asella, Ethiopia, Tel: +251 9102867 66; Email: addansa12@ gmail.com

\section{Review Article \\ Volume 6 Issue 1}

Received Date: December 14, 2020

Published Date: January 25, 2021

DOI: $10.23880 /$ doij-16000237

\section{Abstract}

There is a considerable increase in the proportion of diabetic mellitus (DM) globally. The prevalence of Type 2 DM (T2DM) is increasing across the all over the world. The consumption of alcohol is also rising globally including among the T2DM individuals. Alcohol drinking, principally the heavier consuming is a significant risk factor for many health problems and, thus, it is a main contributor to the global burden of disease. Therefore, the present review article was intended to investigate the effects of alcohol consumption among T2DM individuals which mainly focused on its effect on glycemic control, cardiovascular disease (CVD), mortality associated with T2DM. Several studies have found that light to moderate alcohol consumption among T2DM patients have the beneficial effects regarding the glycemic control, cardiovascular disease, mortality associated with T2DM. However, heavy alcohol ingestion among these individuals has the critical adverse effects. As suggestions, advice on alcohol use should largely aim at lowering heavy ingestions among T2DM subjects. These individuals should be advised on decreasing heavy alcohol consumption since the consequences of heavy amount of alcohol consumption has a severe and substantial impacts on T2DM patients. Further, more attention is needed regarding to these critical public issues to avoid the complications associated with the heavy alcohol consumption among T2DM patients. Health education programs should be created and also any programs that can improve the awareness of these individuals is required to take place in order to improve their health.

Keywords: Mortality; Diabetes Mellitus; Alcohol Consumption; Cardiovascular Disease; T2DM

\section{Introduction}

DM could be occurred due to the defects in insulin secretion, insulin action, or both [1]. In individuals with T2DM, the body doesn't respond appropriately to the insulin [2]. In T2DM, glucose metabolism is normally controlled by a feedback loop which comprises islet $\beta$ cells and insulinsensitive tissues whilst tissue sensitivity to insulin affects the magnitude of $\beta$-cell response. The $\beta$ cells maintain normal glucose tolerance by increasing insulin output if insulin resistance is present. But when $\beta$ cells cannot release sufficient insulin in the presence of insulin resistance, the glucose concentrations will be increased [3]. The impaired insulin secretion and enlarged insulin resistance are the significant pathophysiological features of T2DM [4].

The magnitude of obesity and DM continuous to rise among U.S. adults [5]. DM epidemic was developed in the time of 20th century and remains into the 21st century [6]. The global proportion of DM has enlarged from $4.7 \%$ in 1980 to $8.5 \%$ in 2014 [7]. Its prevalence was likely to be $8.8 \%$ and this was predicted to grow to $10.4 \%$ in 2040 [8] while $8.8 \%$ of the world population was diagnosed with T2DM in 2017 , with the expectation to grow to $9.9 \%$ by 2045 globally [9]. The proportion of subjects with T2DM is growing in developed and developing countries [10]. T2DM and itsassociated complications lead to a major worldwide public health issues by affecting approximately all populations in 


\section{Diabetes \& Obesity International Journal}

both economically developed and developing countries [11].

Alcohol ingestion is a major risk factor for worldwide disease load and causes momentous health loss [12]. The high amount of drinking is a noticeable risk factor for several health problems. So that, it is a major contributor to the worldwide impact of the disease [13]. More than $40 \%$ of the world's adult people consume alcohol globally [14]. Alcohol offers about 4 to $6 \%$ of the average energy use in most Western countries [15]. Overall, alcohol drinking has both adverse and beneficial effects on survival [16]. But, moderate dose of alcohol drinking is linked to a self-perception of good health [17]. The alcohol consumption is less recognized in populations with diabetes relative to the general community [18].

\section{The Effect of Alcohol use in Type 2 Diabetic Subjects}

\section{Glycemia}

The study found that the moderate amount of alcohol consumption helps in glycemic control $[15,19,20]$. Diabetes subjects, alcohol ingesting is inversely associated with glycemic control. Whilst the glycemic control influences an incidence of diabetes complications, the lower A1C levels associated with moderate amount of alcohol drinking where this may reflect the lower risk for complications [19]. The study showed that a moderate amount of alcohol can be consumed with a meal without causing hypoglycaemia in non-insulin-dependent diabetic individuals [15].

A systematic review and meta-analysis of randomized trials that consumption of light to moderate amounts of alcohol, with or without a meal, influence any measure of glycaemic control in individuals with T2DM. These mean that subjects with diabetes do not need to refrain from ingesting moderate amount of alcohol, it does not need to be changed [21]. The meta-analysis finding showed that moderate wine ingestion among T2DM individuals could decrease the level of diastolic blood pressure (DBP) and total cholesterol (TC), but not glucose parameters [22]. Besides, the study showed that heavy alcohol drinkers are at risk for suboptimal longterm weight loss. Whereas, reducing alcohol use may improve weight management among subjects with T2DM [23]. Acute alcohol ingestion improves insulin action by rising insulin sensitivity without influencing beta-cell secretion. This effect might be partially because of the inhibitory effect of alcohol on lipolysis [24]. Besides, the study also revealed that the acute alcohol consumption rises endothelium-dependent brachial artery vasodilation in T2DM and insulin sensitivity [25]. Also, the red wine drinking for two weeks noticeably attenuates insulin-resistance in T2DM subjects without affecting vascular reactivity and nitric oxide production [26].
However, the evidence showed that adults with diabetes who report moderate or heavy alcohol use may be at risk for adverse diabetes outcomes because of suboptimal preventive practices [27]. Alcohol use by diabetics can worsen blood glucose control in these individuals. For instance, chronic alcohol consumptions in well-nourished diabetics can lead to in extreme blood glucose levels while it leads to dangerously low blood glucose levels in patients who are not adequately nourished. Further, heavy use of alcohol in these subjects also can cause the accumulation of certain acids in the blood that may result in severe health outcomes. Added, alcohol use can worsen diabetes-related medical complications [2].

\section{Cardiovascular Disease}

Several studies have been showed that light to moderate alcohol consumption reduces the risk of CVDs [28-33]. Ajani, et al. found that light to moderate amount of alcohol drinking is associated with similar risk reductions in coronary heart disease (CHD) among diabetic and nondiabetic men individuals [28]. The meta-analysis showed that moderate alcohol consumption is associated with a lower risk of CHD in T2DM populations [29]. According to the study findings though potential risks of alcohol intake must be considered, moderate amount of alcohol intake is associated with decreased CHD risk in women with diabetes and should not be routinely discouraged from drinking it [30]. The other study also showed that moderate alcohol intake is associated with the lower risk of CHD in men with T2DM [34].

The moderate wine consumption improved cardiometabolic factors in alcohol-abstinent adults with T2DM and a healthy diet compared with mineral water [35]. A study showed that a moderate red wine consumption among well-controlled T2DM as part of a healthy diet is seemingly safe and modestly decreases cardio-metabolic risk [31]. In T2DM patients, moderate amount of red wine use with meals, significantly decreases oxidative stress and proinflammatory cytokines as well as improving cardiac function after myocardial infarction. Which means it may have a beneficial effect in avoiding of cardiovascular complications in these individuals [36].

In well controlled T2DM subjects $24-31 \mathrm{~g}$ alcohol/day (about 2 to 3 standard drinks) increases awake BP and a 24hour heart rate and lowers asleep BP. However, it does not adversely modify cardiovascular risk factors [37]. Alcohol consumption is a significant prognosticator of coronary events. Low-to moderate ingestion appears to be linked with a decrease in the proportion of acute coronary syndromes in diabetes, whereas heavy ingestion is related to an increase in lipids and BP levels, and risk of acute coronary syndromes occurrence [38]. An evidence from a survey-based crosssectional study indicated high alcohol ingestion in men with 


\section{Diabetes \& Obesity International Journal}

diabetes associated to a lower incidence of cardiovascular events [39]. It is a light alcohol consumption not the high ingestions that has preventive effects on atherosclerosis in T2DM patients [40].

However, diabetes might be the outcome of excessive alcohol drinking which could lead to insulin resistance, then to coronary heart disease [41]. Also, the high amount of alcohol ingestion facilitates the occurrences of atherosclerosis exclusively in diabetic individuals [42]. Heavy amount of alcohol intake in patients with type T2DM is associated to augmented risk of diabetes-related microvascular and macrovascular complications [43].

\section{Mortality}

The evidence suggested that the moderate alcohol using is associated with a decreased risk of premature mortality in subjects with DM $[32,33]$. The evidence showed that the countries that consume primarily beer have greater diabetes mortality rates than countries that consume primarily spirits or wine [44]. The meta-analysis showed that moderate alcohol consumption is associated with a lower risk of mortality in T2DM populations [45]. Among people with diabetes, lightto-moderate alcohol consumption reduces the risks of CVD and all-cause mortality [46]. In light-to-moderate alcohol consumers, the higher serum dehydroepiandrosterone (DHEA)-S concentrations may indicate part of the link between light-to-moderate alcohol consumption and lower CVD mortality [47].

Alcohol consumption in men with glucose intolerance was linked with an important decrease in cardiovascular and all-heart disease mortality [48]. A population-based prospective cohort study findings showed that alcohol ingestion was found to have a beneficial effect in reducing the risk of death because of CHD in individuals with older onset diabetes [49]. The moderate alcohol consumption slightly reduced the mortality among middle-aged and elderly population. But its benefit is depended on age and background of cardiovascular risk [16].

However, Diem et al. found that in T2DM subjects even though moderate alcohol use of 16 to $30 \mathrm{~g}$ per day was related with decreased mortality from CHD and from all causes, alcohol consumption above $30 \mathrm{~g}$ per day was linked with a tendency towards augmented all-cause mortality [50]. Further, the study showed that the raised mortality risk among non-alcohol drinkers seemed to be affected by their past alcohol ingestion rather than their recent abstinence [32]. Lastly, the study suggests that in individuals with T2DM, alcohol consumption should not be discouraged from using alcohol in moderation [51].

\section{Conclusion}

The proportion of DM is increasing globally. As a type of DM, the prevalence of T2DM is growing substantially. This has a critical burden on the individuals with this disease, and also on the government as whole. The present review has found that light to moderate alcohol consumption among T2DM patients has been found to have beneficial effects regarding the glycemic control, cardiovascular disease, mortality associated with T2DM. However, heavy alcohol ingestion among these individuals has the critical adverse effects.

Finally, as suggestions, advice on alcohol use should largely aim at lowering heavy ingestions among T2DM subjects. These individuals should be advised on decreasing heavy alcohol consumption since the consequences of heavy amount of alcohol consumption have severe and substantial impacts on T2DM patients.

\section{Funding}

There was no source of fund for this work.

\section{Disclosure}

The author declares no conflicts of interest in this work.

\section{Author's Contribution}

The author made a significant contribution to conception and design, acquisition of data, or analysis and interpretation of data; took part in drafting, revising or critically reviewing the article; gave final approval of the version to be published; and agree to be accountable for all aspects of the work.

\section{References}

1. The Expert Committee on the Diagnosis and Classification of Diabetes Mellitus (1997) Report of the Expert Committee on the Diagnosis and Classification of Diabetes Mellitus. Diabetes Care 20(7): 1183-1197.

2. Emanuele NV, Swade TF, Emanuele MA (1998) Consequences of Alcohol Use in Diabetics. Alcohol Health Res World 22(3): 211-219.

3. Kahn SE, Cooper ME, Del Prato S (2014) Pathophysiology and treatment of type 2 diabetes: perspectives on the past, present, and future. The Lancet 383(9922): 10681083.

4. Kaku K (2010) Pathophysiology of Type 2 Diabetes and its Treatment Policy. Japan Medical Association Journal 53(1): 41-46. 


\section{Diabetes \& Obesity International Journal}

5. Mokdad AH (2001) The Continuing Epidemics of Obesity and Diabetes in the United States. JAMA 286(10): 11951200.

6. Engelgau MM, Geiss LS, Saaddine JB, Boyle JP, Benjamin SM, et al. (2004) The Evolving Diabetes Burden in the United States. Ann Intern Med 140(11): 945-950.

7. Henning RJ (2018) Type-2 diabetes mellitus and cardiovascular disease. Future Cardiology 14(6): 491509.

8. Ogurtsova K, da Rocha Fernandes JD, Huang Y, Linnenkamp U, Guariguata L, et al. (2017) IDF Diabetes Atlas: Global estimates for the prevalence of diabetes for 2015 and 2040. Diabetes Research and Clinical Practice 128: $40-50$.

9. Standl E, Khunti K, Hansen TB, Schnell O (2019) The global epidemics of diabetes in the 21st century: Current situation and perspectives. Eur J Prev Cardiolog 26(S2): 7-14.

10. van Dierena $S$, Beulensa JWJ, van der Schouwa YT, Grobbeea DE, Nealb B (2010) The global burden of diabetes and its complications: an emerging pandemic. European Journal of Cardiovascular Prevention and Rehabilitation 17(S1): S3-S8.

11. Wu Y, Ding Y, Tanaka Y, Zhang W (2004) Risk Factors Contributing to Type 2 Diabetes and Recent Advances in the Treatment and Prevention. Int J Med Sci 11(11): 1185-2000.

12. Griswold MG, Fullman N, Hawley C, Arian N, Zimsen SRM, et al. (2018) Alcohol use and burden for 195 countries and territories, 1990-2016: a systematic analysis for the Global Burden of Disease Study 2016. The Lancet 392(10152): 1015-1035.

13. Rehm J (2011) The Risks Associated With Alcohol Use and Alcoholism. Alcohol Res Health 34(2): 135-143.

14. Shield KD, Rylett M, Gmel G, Gmel G, Kehoe-Chan TAK, et al. (2013) Global alcohol exposure estimates by country, territory and region for 2005-a contribution to the Comparative Risk Assessment for the 2010 Global Burden of Disease Study: Global alcohol exposure for 2005. Addiction 108(5): 912-922.

15. Christiansen C, Thomsen C, Rasmussen O, Hauerslev C, Balle M, et al. (1994) Effect of alcohol on glucose, insulin, free fatty acid and triacylglycerol responses to a light meal in non-insulin-dependent diabetic subjects. $\mathrm{Br} \mathrm{J}$ Nutr 71(3): 449-454.

16. Thun MJ, Peto R, Lopez AD, Monaco JH, Henley SJ, et al. (1997) Alcohol Consumption and Mortality among Middle-Aged and Elderly U.S. Adults. N Engl J Med 337(24): 1705-1714.

17. Poikotainen K, Vartiainen E, Korhonen HJ (1996) Alcohol intake and Subjective Health. American Journal of Epidemiology 144(4): 346-350.

18. Polsky S, Akturk HK (2017) Alcohol Consumption, Diabetes Risk, and Cardiovascular Disease Within Diabetes. Curr Diab Rep 17(12): 136.

19. Ahmed AT, Karter AJ, Warton EM, Doan JU, Weisner CM (2008) The Relationship Between Alcohol Consumption and Glycemic Control Among Patients with Diabetes: The Kaiser Permanente Northern California Diabetes Registry. J GEN INTERN MED 23(3): 275-282.

20. Shai I, Wainstein J, Harman-Boehm I, Raz I, Fraser D, et al. (2007) Glycemic Effects of Moderate Alcohol Intake Among Patients With Type 2 Diabetes: A multicenter, randomized, clinical intervention trial. Diabetes Care 30(12): 3011-3016.

21. Hirst JA, Aronson JK, Feakins BG, Ma C, Farmer AJ, et al. (2017) Short- and medium-term effects of light to moderate alcohol intake on glycaemic control in diabetes mellitus: a systematic review and meta-analysis of randomized trials. Diabet Med 34(5): 604-611.

22. Ye J, Chen X, Bao L (2019) Effects of wine on blood pressure, glucose parameters, and lipid profile in type 2 diabetes mellitus: A meta-analysis of randomized interventional trials (PRISMA Compliant). Medicine 98(23): e15771.

23. Chao AM, Wadden TA, Tronieri JS, Berkowitz RI (2019) Alcohol Intake and Weight Loss During Intensive Lifestyle Intervention for Adults with Overweight or Obesity and Diabetes. Obesity 27(1): 30-40.

24. Avogaro A, Watanabe RM, Dall'Arche A, Vigili De Kreutzenberg S, Tiengo A, et al. (2004) Acute Alcohol Consumption Improves Insulin Action Without Affecting Insulin Secretion in Type 2 Diabetic Subjects. Diabetes Care 27(6): 1369-1374.

25. Schaller G, Kretschmer S, Gouya G, Haider DG, Mittermayer F, et al. (2009) Alcohol Acutely Increases Vascular Reactivity together with Insulin Sensitivity in Type 2 Diabetic Men. Exp Clin Endocrinol Diabetes 118(1): 57-60.

26. Napoli R, Cozzolino D, Guardasole V, Angelini V, Zarra E, et al. (2005) Red wine consumption improves insulin resistance but not endothelial function in type 2 diabetic 


\section{Diabetes \& Obesity International Journal}

patients. Metabolism 54(3): 306-313.

27. Chew LD, Nelson KM, Young BA, Bradley KA (2005) Association between alcohol consumption and diabetes preventive practices. Fam Med 37(8): 589-594.

28. Ajani UA, Gaziano JM, Lotufo PA, Liu S, Hennekens CH, et al. (2000) Alcohol Consumption and Risk of Coronary Heart Disease by Diabetes Status. Circulation 102(5): 500-505.

29. Koppes LLJ, Dekker JM, Hendriks HFJ, Bouter LM, Heine RJ (2006) Meta-analysis of the relationship between alcohol consumption and coronary heart disease and mortality in type 2 diabetic patients. Diabetologia 49(4): 648-652.

30. Solomon CG, Hu FB, Stampfer MJ, Colditz GA, Speizer FE, et al. (2000) Moderate Alcohol Consumption and Risk of Coronary Heart Disease Among Women With Type 2 Diabetes Mellitus. Circulation 102(5): 494-499.

31. Gepner Y, Golan R, Harman-Boehm I, Henkin Y, Schwarzfuchs D, et al. (2015) Effects of Initiating Moderate Alcohol Intake on Cardiometabolic Risk in Adults With Type 2 Diabetes: A 2-Year Randomized, Controlled Trial. Ann Intern Med 163(8): 569-579.

32. Sluik D, Boeing H, Bergmann MM, Schütze $M$, Teucher B, et al. (2012) Alcohol consumption and mortality in individuals with diabetes mellitus. Br J Nutr 108(7): 1307-1315.

33. Blomster JI, Zoungas S, Chalmers J, Li Q, Chow CK, et al. (2014) The Relationship Between Alcohol Consumption and Vascular Complications and Mortality in Individuals With Type 2 Diabetes. Dia Care 37(5): 1353-1359.

34. Tanasescu M, Hu FB, Willett WC, Stampfer MJ, Rimm EB (2001) Alcohol consumption and risk of coronary heart disease among men with type 2 diabetes mellitus. Journal of the American College of Cardiology 38(7): 1836-1842.

35. Jain M, Wernick R (2016) In abstinent adults with type 2 diabetes, a daily glass of wine (vs mineral water) improved cardiometabolic factors. Ann Intern Med 164(4): JC17.

36. Marfella R, Cacciapuoti F, Siniscalchi M, Sasso FC, Marchese F, et al. (2006) Effect of moderate red wine intake on cardiac prognosis after recent acute myocardial infarction of subjects with Type 2 diabetes mellitus. Diabetic Med 23(9): 974-981.

37. Mori TA, Burke V, Zilkens RR, Hodgson JM, Beilin LJ, et al. (2016) The effects of alcohol on ambulatory blood pressure and other cardiovascular risk factors in type 2 diabetes: a randomized intervention. Journal of Hypertension 34(3): 421-428.

38. Pitsavos C, Makrilakis K, Panagiotakos DB, Chrysohoou C, Ioannidis I, et al. (2005) The J-shape effect of alcohol intake on the risk of developing acute coronary syndromes in diabetic subjects: the CARDIO2000 II Study. Diabet Med 22(3): 243-248.

39. Jakobsen ML, Larsen JR, Glümer C, Juel K, Ekholm O, et al. (2016) Alcohol consumption among patients with diabetes: a survey-based cross-sectional study of Danish adults with diabetes. Scand J Public Health 44(5): 517524.

40. Wakabayashi I, Kobaba-Wakabayashi R, Masuda H (2002) Relation of Drinking Alcohol to Atherosclerotic Risk in Type 2 Diabetes. Diabetes Care 25(7): 1223-1228.

41. Balkau B, Eschwege E, Fontbonne A, Claude JR, Warnet JM (1992) Cardiovascular and alcohol-related deaths in abnormal glucose tolerant and diabetic subjects. Diabetologia 35(1): 39-44.

42. Sakuta H, Suzuki T, Katayama Y, Yasuda H, Ito T (2005) Heavy alcohol intake, homocysteine and Type 2 diabetes. Diabet Med 22(10): 1359-1363.

43. Elgendy R, Deschênes SS, Burns RJ, Levy M, Schmitz N (2019) Alcohol consumption, depressive symptoms, and the incidence of diabetes-related complications. Journal of Diabetes 11(1): 14-22.

44. Keilman PA (1983) Alcohol consumption and diabetes mellitus mortality in different countries. Am J Public Health 73(11): 1316-1317.

45. Heianza Y, Arase Y, Saito K, Tsuji H, Fujihara K, et al. (2013) Role of alcohol drinking pattern in type 2 diabetes in Japanese men: the Toranomon Hospital Health Management Center Study 11 (TOPICS 11). The American Journal of Clinical Nutrition 97(3): 561-568.

46. Ley SH, Sun Q, Jimenez MC, Rexrode KM, Manson JE, et al. (2014) Association between alcohol consumption and plasma fetuin-A and its contribution to incident type 2 diabetes in women. Diabetologia 57(1): 93-101.

47. Fukui M, Kitagawa $\mathrm{Y}$, Nakamura N, Kadono M, Hasegawa G, et al. (2005) Association between alcohol consumption and serum dehydroepiandrosterone sulphate concentration in men with Type 2 diabetes: a link to decreased cardiovascular risk. Diabet Med 22(10): 1446-1450.

48. Nakamura Y, Ueshima H, Kadota A, Hozawa A, Okamura 
Diabetes \& Obesity International Journal

T, et al. (2009) Alcohol intake and 19-year mortality in diabetic men: NIPPON DATA80. Alcohol 43(8): 635-641.

49. Valmadrid CT (1999) Alcohol Intake and the Risk of Coronary Heart Disease Mortality in Persons With OlderOnset Diabetes Mellitus. JAMA 282(3): 239-246.

50. Diem P, Deplazes M, Fajfr R, Bearth A, Muller B, et al.
(2003) Effects of alcohol consumption on mortality in patients with Type 2 diabetes mellitus. Diabetologia 46(11): 1581-1585.

51. Bantle AE, Thomas W, Bantle JP (2008) Metabolic effects of alcohol in the form of wine in persons with type 2 diabetes mellitus. Metabolism 57(2): 241-245. 